\title{
Broadband Circularly Polarized Embedded DRA Array for WLAN Applications
}

\author{
Aiswarya Raj \\ PG Scholar \\ Department of Electronics and Communication \\ Engineering \\ TKM College of Engineering Kollam, India
}

\author{
Dr. Sheeba O \\ Professor (HOD) \\ Department of Electronics and Communication \\ Engineering \\ TKM College of Engineering Kollam, India
}

\begin{abstract}
In this paper, a dielectric resonator antenna array is proposed. A broadband circularly polarized half-split embedded cylindrical dielectric resonator antenna with varying permittivity along the radial direction is taken as a single radiating element for a two elemental array configuration developed for the commercial WLAN applications. The proposed antenna array consists of two embedded half cylindrical structures (DRAs). Each embedded structure is a combination of two DRAs of different permittivity along radial direction. The DRA with lower permittivity embedded in the groove of DRA with higher permittivity. A modified coplanar waveguide feed is used to excite the array structure thus the ground plane is modeled over the substrate. By triggering orthogonal modes in CDRA through the feed network, generates circular polarization in the braodside direction and is confirmed by the rotation of electric field lines. The proposed array configuration will shows a significant bandwidth greater than $550 \mathrm{MHz}$ with central frequency of $5.25 \mathrm{GHz}$. The maximum gain in broadside direction is found to be $3.99 \mathrm{~dB}$.
\end{abstract}

Keywords - Broadband, circular polarization, coplanar waveguide feed, dielectric resonator.

\section{INTRODUCTION}

In wireless communication scenario, dielectric resonators have become better choice over microstrip patch antennas due to its flexibility in antenna feeding networks and immense structural diversity. The DRA consist of high dielectric constant materials and high quality factors, thus it offers low metallic loss, bandwidth enhancement and high radiation efficiency. Moreover, numerous design solutions are existing for improving the gain and radiation properties of DRAs. Hence, it become attractive hand for antenna designers.

Many applications such as WLAN, WiMAX, radar applications and video conferencing require broadband operation. So DRA grabs more attraction to form a resonator. Systems with circular polarization are insensitive to antenna (transmitting and receiving) configurations. Also, they are susceptible to propagation effects, weather altrations and interferences. Thus, designers focused more over circularly polarized DRAs for wireless applications.

In this paper, circular polarization is realized by generating orthogonal modes in CDRAs. A modified coplanar waveguide feed is considered since it reduces dispersion effects and radiation losses. A single HSCDRA doesn't show significant amount of gain hence array designing is selected. The array structure combine the signlas from individual resonators inorder to achieve improved performance as compared with a single antenna. This reduce interference and is more sensitive in a particular direction thus maximise the siganl to interference noise ratio (SINR). Near/far field antenna measurements such as reflection coefficient, axial ratio, radiation pattern, radiation efficiency and gain were simulated and anlaysed using ANSOFT-HFSS software.

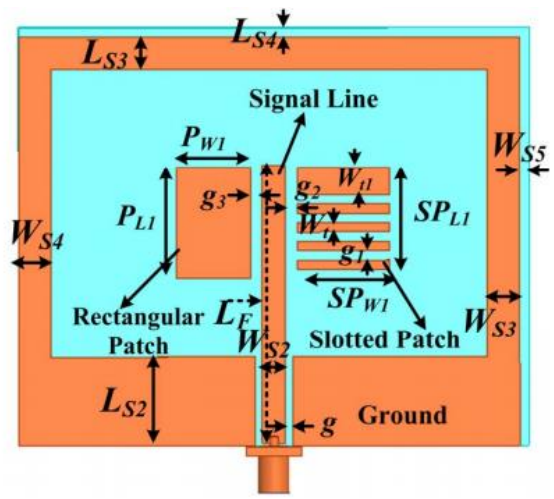

(a)

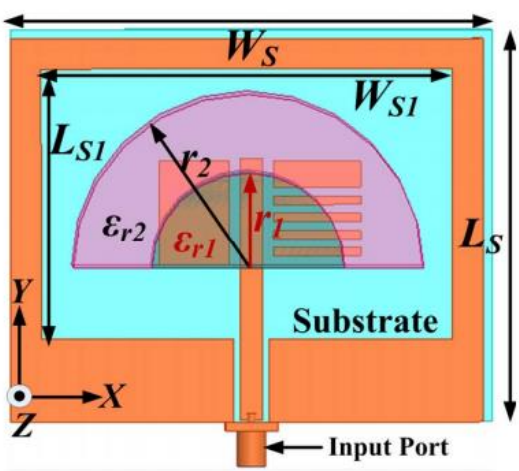

(b)

Fig. 1: Geometry of proposed antenna (a) top view modified CPW-fed (b) top view HS-CDRA 


\section{ANTENNA CONFIGURATION}

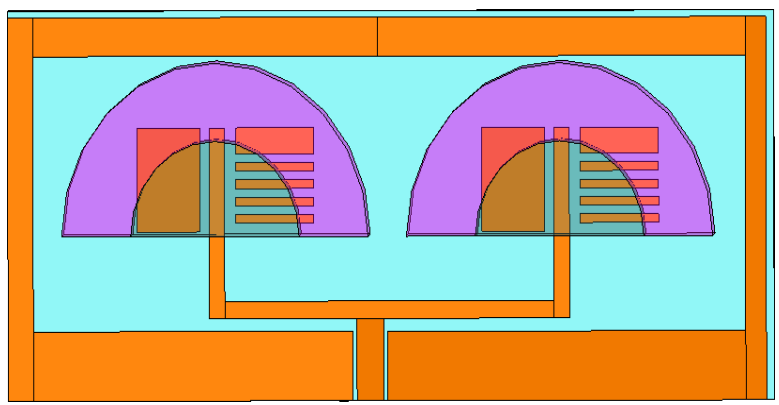

Fig 2: Geometry of 2x1 array

Fig.2 depicts the geometry of proposed broadband circularly polarized DRA array having two embedded elements. Each element consists of half-split CDRA made of two different permittivity along radial direction. The DRA materials are Polyflon Polyguide $(\varepsilon \mathrm{r} 1=2.32, \mathrm{r}=11 \mathrm{~mm})$ and Roger RT/Duroid 6010 ( $\mathrm{Er} 2=10.2, \mathrm{r}=20 \mathrm{~mm})$. DRA elements are glued together using Araldite resin with permittivity of $\varepsilon r=3.6$. The substrate material is FR4 glass epoxy $(\varepsilon r=4.4$, tand $=0.025$, height $=1.6 \mathrm{~mm}$ ). Both the elements are coupled using a centre feed network. Coplanar waveguide feed is usedfor excitation and is realized by a signal line (feeding strip), rectangular shaped slotted and unslotted patches etched on the top of substrate and the ground plane on the same plane. Circular polarization is generated by triggering orthogonal modes (perpendicular modes) in CDRA. Based on the antenna geometry, TM11d mode is excited.

\section{DESIGN MECHANISM}

The design methodology consisdered for proposed antenna array is based on coupling mechanism between cylindrical DRA of different permittivty and centre fed network structure for exciting the array elements through modified coplanar waveguide feed. The design procedures have been summarized below.

Step 1:

Designing of DRA with CPW feeding. Acylindrical structure is chosen since surface waves are less in quantity and is easily modified. Here, focused on HE11 mode excitation because we require circular polarization and CPW configuration of mode. HE11 mode is composed of approxiamtely $85 \%$ of TE11 mode and $15 \%$ of TM11. This mode is desirable for the most demanding applications due to its excellent radiation properties. The dimension of DRA is proportional to wavelength and is inversely proportional to the square root of dielectric permittivity. The radius and height are the tuning parameters of cylindrical DRA. The resonant frequency,

$$
\begin{gathered}
F=0.208 * \frac{C}{2 \prod h \sqrt{\varepsilon_{r}}+1}\left[1+0.7013\left(\frac{r}{h}\right)-0.002713\left(\frac{r}{h}\right)^{2}\right] \\
\left\{0<\frac{r}{h}<6.1\right\}
\end{gathered}
$$

where $\varepsilon r=$ sum of permittivities of two DRAs.

Step 2:

Inserting embedded DRA structure. Efficient coupling mechanism should be adopted.

\section{Step 3:}

Apply patch inside embedded DRA and create symmetrical patch with bilateral symmetry.Calculation of patch parameters by surface modulation similar to normal patch design equations with additional constraint of $1<\mathrm{L} / \mathrm{W}<1.5$.

Step 4:

Apply slot structure to patch plane in embedded structure. Number of slots $=$ width of slot divided by total width of patch. Slots are inserted in left hand side patch for obtaining right hand circular polarization. The dimension of slot is calculated by using the equations provided by Babinet's Principle for $\lambda / 4$.

\section{Step 5:}

Design and implement array configuration. A two elemental DRA array is to be designed and simulated.

Distance between array elements, $\lambda \mathrm{g}=\frac{c}{f_{0} \sqrt{\varepsilon_{r}}}$

Feed point positions, $\mathrm{Xf}=\frac{L}{\sqrt{2 \varepsilon_{e f f} L}}, \mathrm{Yf}=\frac{W}{2}$

Width of feeding strip, $\mathrm{W}=\ell^{\frac{7.48 h}{\frac{Z_{0} \sqrt{\varepsilon_{r}+1.41}}{87}}}$,

$\mathrm{ZO}$ is the impedance parameter.

\section{STRUCTURAL ANALYSIS}

\section{A. Effect of embedded structure}

Fig.3 show the effect of embedded structure on input reflection coefficient and axial ratio. The input reflection coefficient decreases with embedded structure (DRA with lower permittivity inserted in the groove of DRA with higher permittivity). This is due to the coupling between DRAs. Value of axial ratio reflects that embedded structure generates circular polarization(Axial Ratio $<6 \mathrm{~dB}$ ). 


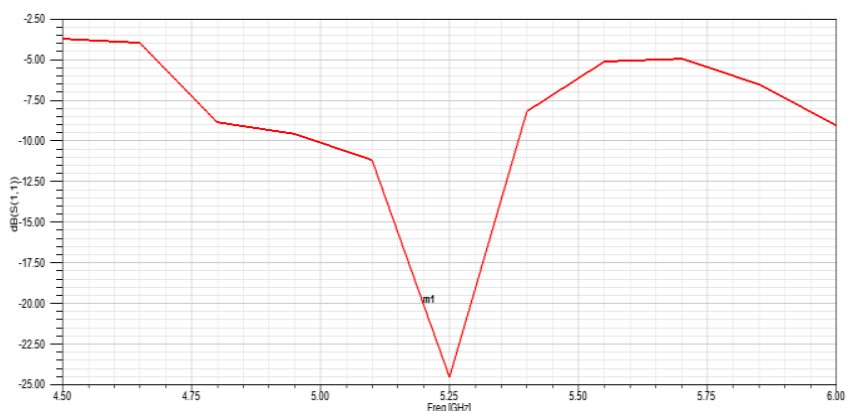

(a) Input reflection coefficient (embedded DRA)

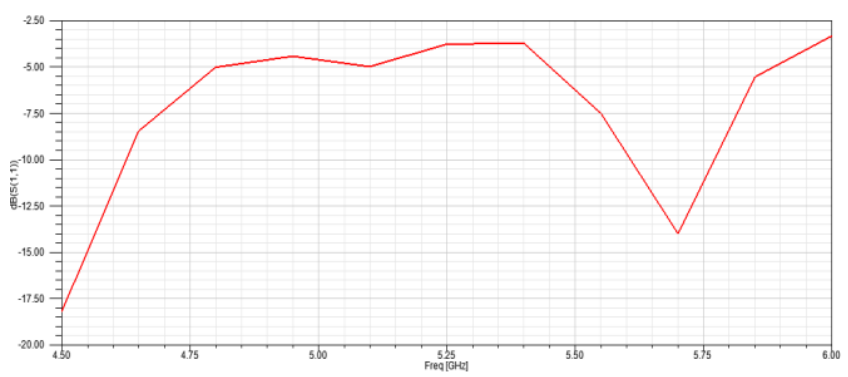

(b) Input reflection coefficient (single DRA)

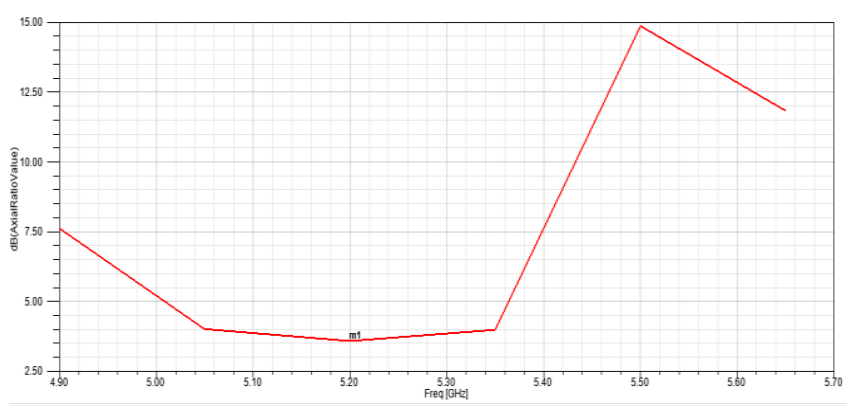

c) Axial ratio bandwidth (embedded DRA)

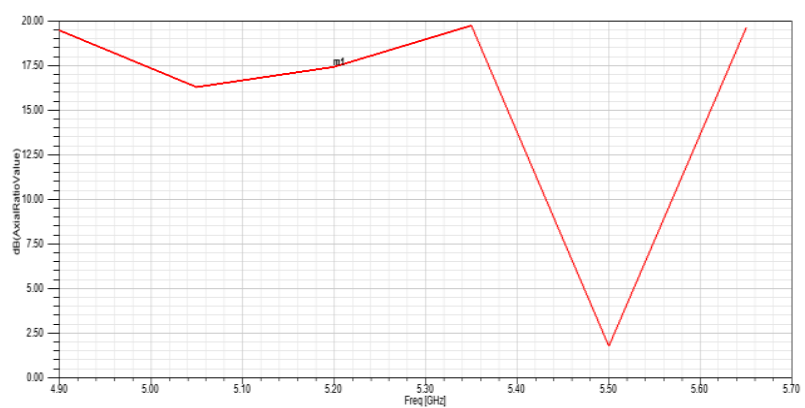

(d) Axial ratio bandwidth (single DRA)

Fig 3: Effect of embedded structure on proposed array

\section{B. Effect of slotted patch}

Fig.4 show the effect of slotted patch over input reflection coefficient and working bandwidth. Single DRA structure exhibits high reflection losses than an embedded structure. Working band enhanced from $300 \mathrm{MHz}$ to $550 \mathrm{MHz}$ by the insertion of slotted patch, so proposed antenna exhibits broadband operation. Axial ratio and radiation pattern reflects the generation of right hand circular polarization due to slotted patch on left side of embedded DRA.

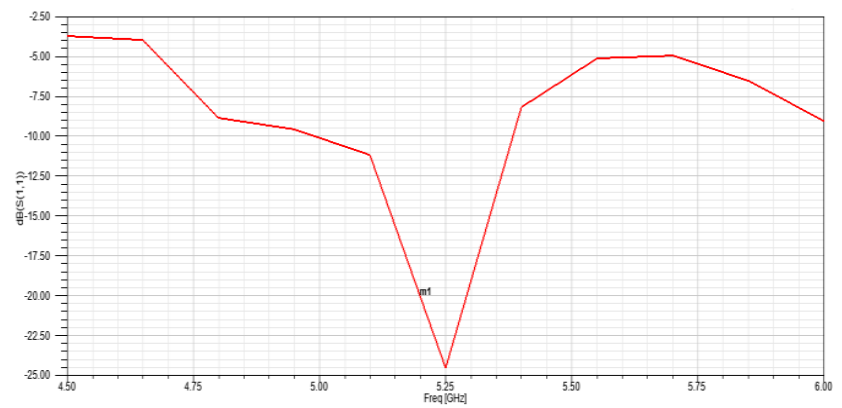

(a) Input reflection coefficient (slotted patch)

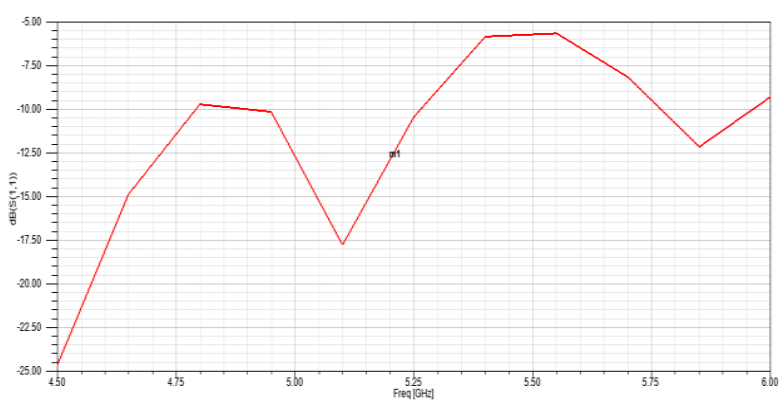

(b) Input reflection coefficient (unslotted patch)

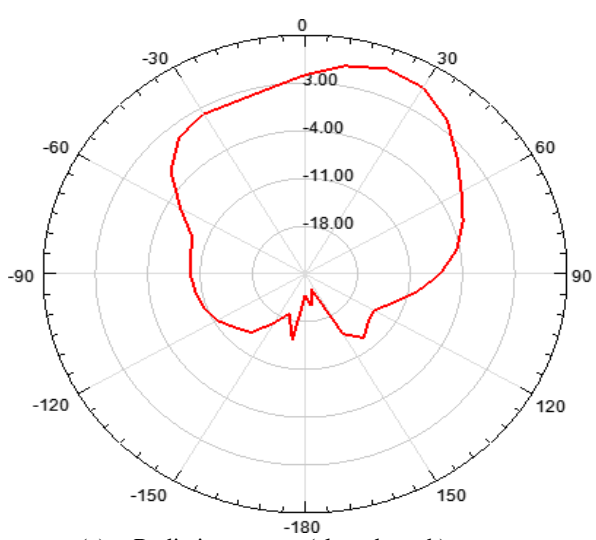

(c) Radiation pattern (slotted patch)

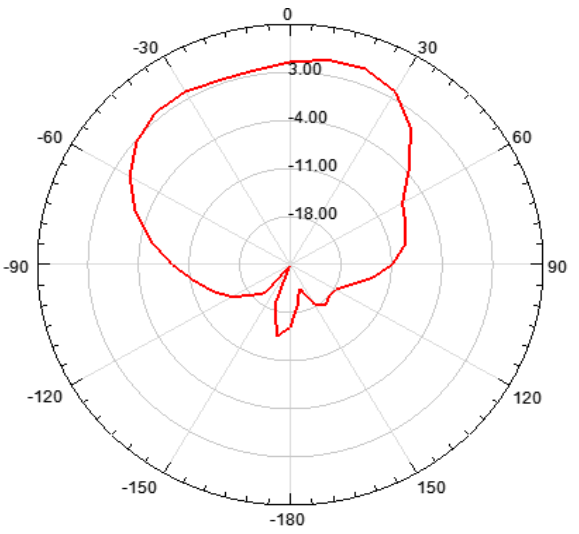

(d) Radiation pattern (unslotted patch)

Fig 4: Effect of slotted structure on proposed array 


\section{SIMULATED RESULTS}

The prototype of antenna array has been designed and simulated in HFSS software. Various antenna parameters are simulated and measured.It is observed that better results are obtained using two elemental array rather than a single element. Figure depicts the input reflection coefficient where, S11<-10dB (-24.5dB) input impedance bandwidth has been found to be $4.85-5.37 \mathrm{GHz}$. Axial Ratio bandwidth has been shown in figure, it is found to be $3.6 \mathrm{~dB}$ in central frequency $5.25 \mathrm{GHz}$. The working band of array is about $550 \mathrm{MHz}$ reflects a broadband operation. The average gain of proposed array in broadside direction has been found that $4 \mathrm{~dB}$ (4.8$5.45 \mathrm{GHz}$ ) as shown in figure. Figure illustrates the right hand circular polarization radiation pattern of a proposed array.

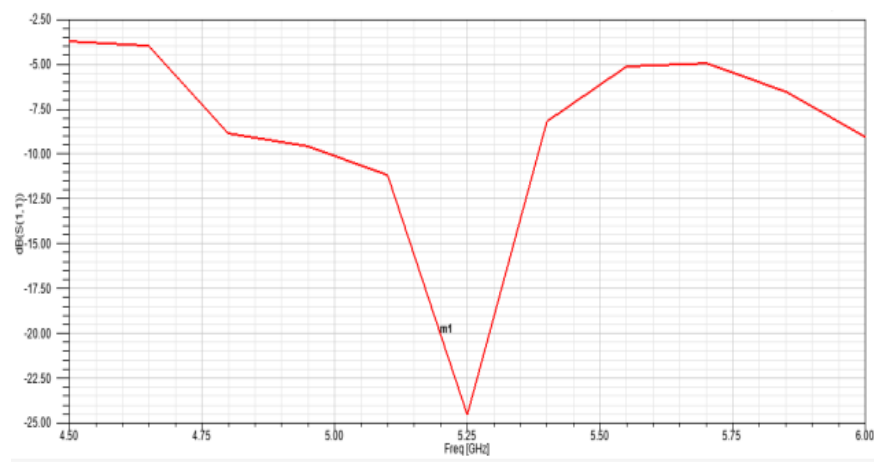

Fig 5: Input reflection coefficient of proposed array

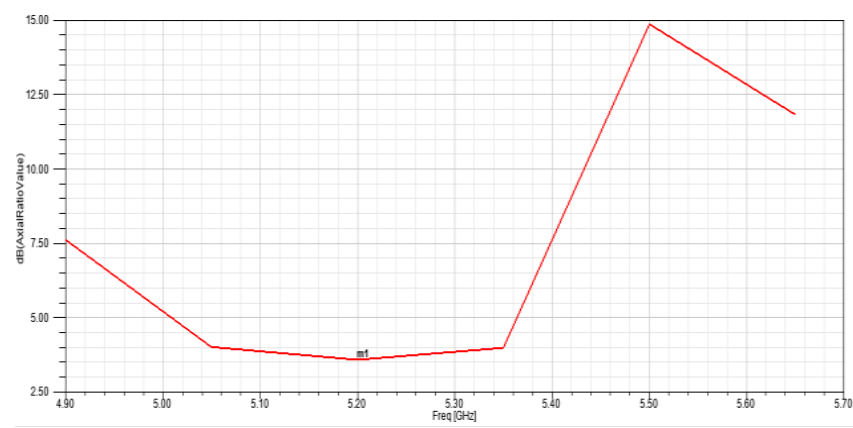

Fig 6: Axial ratio bandwidth of proposed array

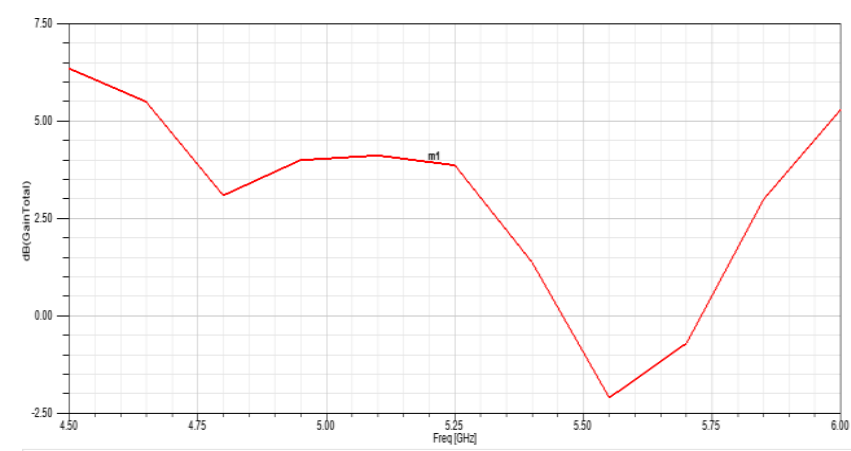

Fig 7: Gain of proposed array

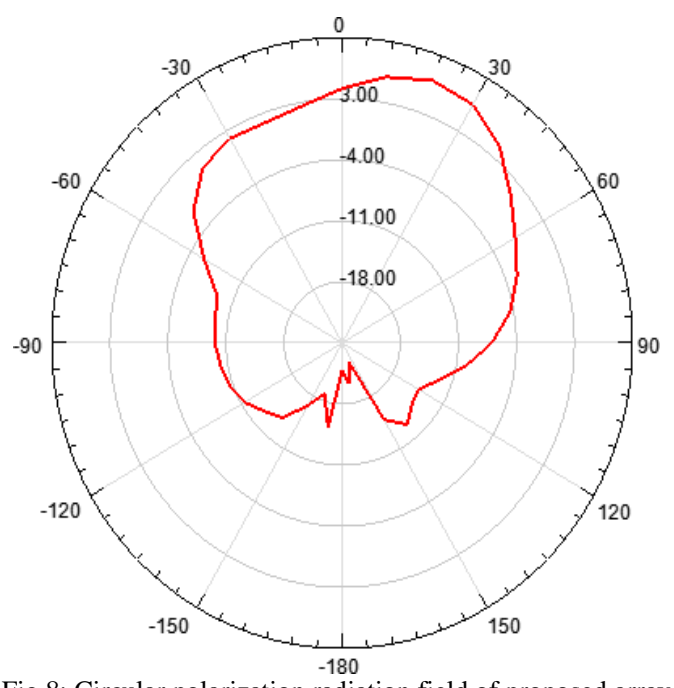

Fig 8: Circular polarization radiation field of proposed array

\section{CONCLUSION}

A broadband circularly polarized half split embedded cylindrical DRA (HS-CDRA) array has been designed and simulated. Proposed antenna array is excited by a modified coplanar waveguide feed with a centre fed network configuration.HE11 mode is excited inside HS-CDRA to realize circular polarization. Designed array can obtain S11 $<-10 \mathrm{~dB}$ input impedance bandwidth of $500 \mathrm{MHz}$ (centered on $5.25 \mathrm{GHz}$ ) and $\mathrm{AR}$ of $3.6 \mathrm{~dB}$ (centered on $5.25 \mathrm{GHz}$ ). The array configuration provides very low radiation losses, better gain and broadband working.

\section{AKNOWLEDGEMENT}

At the commencement, I realize it my obligation to thank the Almighty God for giving me necessary wisdom to complete this work successfully. I express my profound gratitude to our head of the department and my project guide Dr. Sheeba O for giving me the accessory environment to acquire knowledge and skill. I express my profound gratitude to our PG coordinator, Prof. Abid Hussain Muhammed, for his advice and support for this work. I also express my heartfelt thanks to all my teachers, friends and my parents for providing the much needed support during the paper preparation and presentation of my project.

\section{REFERENCES}

[1] S. Gao, Q. Luo, and F. Zhu, "Circularly polarized antenna," West Sussex, UK, Wiley 2014.

[2] A. Petosa, "Dielectric resonator antenna Handbook," Norwood, MA, USA: Artech House 2007.

[3] R. K. Mongia and P. Bhartia, "Dielectric Resonator Antennas A Review and General Design Relations for Resonant Frequency and Bandwidth," International Journal of Microwave and Millimeter- Wave Computer- Aided Engineering, 4, 3, pp. 230- 247, 1994.

[4] R. K. Chaudhary, R. Kumar, and K. V. Srivastava, "Wideband Ring Dielectric Resonator Antenna with Annular-Shaped Microstrip Feed," IEEE Antennas and Wireless Propag. Lett., Vol. 12, pp. 595-598, 2013.

[5] D. Guha, B. Gupta, C. Kumar, and Y. M. M. Antar, "Segmented Hemispherical DRA: New Geometry Characterized and Investigated in Multi-Element Composite Forms for Wideband Antenna Applications," 
IEEE Trans. on Antennas and Propag., Vol. 60, No. 3, pp. 1605-1610, Mar. 2012.

[6] R. Kumar and R. K. Chaudhary, "Modified Microstrip-line-fed Rectangular Dielectric Resonator Antenna Coupled with Slotted Ground Plane for Wideband Circular Polarization," Microw. and Opt. Te chn. Lett., Vol. 58, no. 1, pp. 206-210, Jan. 2016.

[7] K. W. Leung and H. K. Ng, "Theory and experiment of circularly polarized dielectric resonator antenna with a parasitic patch," IEEE Trans. Antennas Propag., Vol. 51, no. 3, pp. 405- 412, Mar. 2003.

[8] K. W. Leung, W. C. Wong, K. M. Luk, and E. K. N. Yung, "Circularpolarized dielectric resonator antenna excited by dual conformal strips," Electron. Lett., Vol. 36, no. 6, pp. 484_6, Mar. 2000.
[9] R. Kumar and R. K. Chaudhary, "A Wideband Circularly Polarized Cubic Dielectric Resonator Antenna Excited with Modified Microstrip Feed," IEEE Antennas and Wireless Propag. Lett., Vol. 55, pp. 1285 1288, 2016.S.

[10] Fakhte, H. Oraizi and R. Karimian, "A Novel Low-Cost Circularly Polarized Rotated Stacked Dielectric Resonator Antenna," IEEE Antennas and Wireless Propag. Lett., Vol. 13, pp. 722-725, 2014 